\title{
ON SOME SPECTRAL PROPERTIES OF OPERATORS GENERATED BY QUASI-DIFFERENTIAL MULTI-INTERVAL SYSTEMS *
}

\author{
MAKSIM SOKOLOV ${ }^{\dagger}$
}

\begin{abstract}
We construct the common and the ordered spectral representation for operators, generated as direct sums of self-adjoint extensions of quasi-differential minimal operators on a multiinterval set (self-adjoint vector-operators), acting in a Hilbert space. The structure of the ordered representation is investigated for the case of differential coordinate operators. Results, connected with other spectral properties of such vector-operators, such as the introduction of the identity resolution and the spectral multiplicity have also been obtained.

Vector-operators have been mainly studied by W.N. Everitt, L. Markus and A. Zettl. Being a natural continuation of Everitt-Markus-Zettl theory, the presented results reveal the internal structure of self-adjoint differential vector-operators and are essential for the further study of their spectral properties.
\end{abstract}

\section{Preliminaries.}

1.1. Problem overview. In 1985, F. Gesztesy and W. Kirsch published their work [1], where they considered an example of a Schrödinger operator generated by the Hamiltonian

$$
H=-\frac{d^{2}}{d x^{2}}+\left(s^{2}-\frac{1}{4}\right) \frac{1}{\cos ^{2} x}, s>0 .
$$

Since the potential of (1) has a countable number of singularities on $\mathbb{R}$ which spoil the local integrability, they constructed operators $T_{i}$, generated by (1) in the spaces

$$
L^{2}\left(-\frac{\pi}{2}+i \pi, \frac{\pi}{2}+i \pi\right), i \in \mathbb{Z},
$$

and then considered the direct sum operator $\oplus_{i \in \mathbb{Z}} T_{i}$ in the space

$$
\oplus_{i \in \mathbb{Z}} L^{2}\left(-\frac{\pi}{2}+i \pi, \frac{\pi}{2}+i \pi\right)
$$

The work [1] stimulated other researchers to generalize the problem. In [2], W.N. Everitt and the coauthors considered quasi-differential direct sum operators, generated by a countable multi-interval system, with intervals being subsets of one copy of the real line. In 1992, W.N. Everitt and A. Zettl [3] studied direct sums of minimal and maximal operators generated by arbitrary formally self-adjoint expressions in Hilbert spaces considered on arbitrary intervals (maximal and minimal vector-operators). Later in 2000, vector-operators were also considered in complete locally convex spaces by R.R. Ashurov and W.N. Everitt [4], which was a natural generalization of their work [5]. Since 1992, quasi-differential vector-operators have mostly been investigated in connection with their non-spectral theory, such as the introduction of minimal and maximal vector-operators and their relationship (it was shown that the adjoint of a minimal vector-operator is maximal in a Hilbert space [3], and the analogous result with the modification for Frechet spaces was obtained in [4]). A lot of work has been carried out by W.N. Everitt and L. Markus in order to develop the theory of self-adjoint extensions for vector-operators with the employment

\footnotetext{
*Received May 30, 2003; accepted for publication February 6, 2004.

${ }^{\dagger}$ ICTP Affiliated Center, Mechanics and Mathematics Department, National University of Uzbekistan, Uzbekistan, Tashkent 700095 (sokolovmaksim@hotbox.ru).
} 
of symplectic geometry. In connection with this, see their recent memoirs [6] and [7]. Another group of scientists studied differential operators on graphs. In some cases, such theory has a close connection with that developed by W.N. Everitt, L. Markus and A. Zettl since certain boundary conditions may lead to the consideration of a differential operator on a graph as a direct sum operator. Some most modern results in connection with the spectral theory of differential operators on graphs belong to R. Carlson [8, 9] and P. Kurasov, F. Stenberg [10].

The theory of operators generated by multi-interval systems finds its applications in many problems of quantum mechanics, theory of semiconductors and theoretical computer science; good bibliographical references for these subjects may be found in $[7]$.

Since the theory of quasi-differential vector-operators in a Hilbert space is quite young and the most recent studies have concerned mostly problems connected with their common theory, small attention was given to its spectral aspects. Some results, describing the position of spectra of vector-operators were presented in 1985 in [1] and the most recent results belong to Sobhy El-Sayed Ibrahim [11, 12]. Some spectral properties of self-adjoint vector-operators were presented by M.S. Sokolov in [13] and R.R. Ashurov, M.S. Sokolov in [14, 15]. Nevertheless, a rigorous structural spectral theory for such operators has not been developed yet. The present work is designed to make essential steps in this direction. It completely covers abstract results, briefly described in $[13,14]$ with some modifications. It also presents the new results, describing the structure of the ordered spectral representation and eigenfunction expansions.

1.2. Quasi-differential operators and vector-operators. Basic concepts of quasi-differential operators are described in $[3,6]$. A good reference for operators with real coefficients is the book of M.A. Naimark [16].

Let us have a number $n \in \mathbb{N}, n \geqslant 2$, and an arbitrary interval $I \subseteq \mathbb{R}$. Let $Z_{n}(I)$ be a set of Shin-Zettl matrices. These are matrices $A=\left\{a_{r s}\right\}, a_{r s}: I \rightarrow \mathbb{C}$ of the order $n \times n$, such that for almost all $x \in I$ :

$$
\left\{\begin{array}{lll}
(i) \quad a_{r s} \in L_{l o c}(I), & r, s=\overline{1, n} \\
(i i) \quad a_{r, r+1}(x) \neq 0, & r=\overline{1, n-1} \\
(\text { iii }) & a_{r s}=0, & s=\overline{r+2, n} ; r=\overline{1, n-2}
\end{array}\right.
$$

Consider a function $f: I \rightarrow \mathbb{C}$; its quasi-derivatives relatively to a Shin-Zettl matrix $A$ are defined by

$$
\left\{\begin{aligned}
(i) \quad f_{A}^{[0]} & :=f \\
(i i) \quad f_{A}^{[r]} & :=\frac{1}{a_{r, r+1}}\left[\frac{d}{d x} f_{A}^{[r-1]}-\sum_{s=1}^{r} a_{r s} f_{A}^{[s-1]}\right], \quad r=\overline{1, n-1} \\
(\text { iii }) \quad f_{A}^{[n]} & :=\frac{d}{d x} f_{A}^{[n-1]}-\sum_{s=1}^{n} a_{n s} f_{A}^{[s-1]}
\end{aligned}\right.
$$

Let us introduce a linear manifold $D(A) \subset A C_{l o c}(I)$ :

$$
D_{A}(I):=\left\{f: I \rightarrow \mathbb{C} \mid f_{A}^{[r-1]} \in A C_{l o c}(I) \quad(r=\overline{1, n})\right\}
$$

It is possible to see, that $f \in D_{A}(I)$ implies $f_{A}^{[n]} \in L_{l o c}(I)$, and it is possible to prove that $D_{A}(I)$ is dense in $L_{l o c}(I)$. 
Relative to a matrix $A \in Z_{n}(I)$, we have the quasi-differential expression $M_{A}[f]=$ $i^{n} f_{A}^{[n]}, f \in D_{A}(I)$.

The matrix $A^{+} \in Z_{n}(I)$ designates the Lagrange adjoint matrix to $A$ if $A^{+}:=$ $-L_{n}^{-1} A^{*} L_{n}$, where $A^{*}$ is the adjoint matrix, and $L_{n}=\left\{l_{r s}\right\}$ is the $(n \times n)$-matrix, defined as:

$$
l_{r, n+1-r}=\left\{\begin{array}{cl}
(-1)^{r-1}, & r=\overline{1, n} \\
0, & \text { for other } r, s .
\end{array}\right.
$$

Using this notation we suppose that in this work we deal only with Lagrange symmetric (formally self-adjoint) expressions, that is $M_{A^{+}}[f]=M_{A}[f]=\tau(f)$, where $\tau$ is an alternative denotation for a Lagrange symmetric expression.

For a quasi-differential expression $M_{A}[f]$, the Lagrange formula is known $([\alpha, \beta] \subseteq$ $I$ - an arbitrary compact subinterval of $I$ ):

$$
\int_{\alpha}^{\beta}\left\{\overline{g(x)} M_{A}[f](x)-f(x) \overline{M_{A^{+}}[g(x)]}\right\} d x=[f, g]_{A}(\beta)-[f, g]_{A}(\alpha),
$$

where $f \in D_{A}, g \in D_{A^{+}},[f, g]_{A}(\beta)$ and $[f, g]_{A}(\alpha)$ may be derived from:

$$
[f, g]_{A}(x)=i^{n} \sum_{i=1}^{n}(-1)^{i-1} f_{A}^{[i-1]}(x) \overline{g_{A^{+}}^{[n-i]}(x)}, \quad x \in I .
$$

Let $\omega>0$ be a weight function from $L_{l o c}(I), \omega: I \rightarrow \mathbb{R}$; the Hilbert space $L^{2}(I: \omega)$ is formed as usual.

We define maximal and minimal operators as follows:

Definition 1.1. Operators $T_{\max }$ and $T_{\min }$ are called respectively maximal and minimal operators if they are generated by $\tau(f)$ on the domains $D\left(T_{\max }\right)$ and $D\left(T_{\min }\right)$ :

$$
\begin{gathered}
D\left(T_{\text {max }}\right)=\left\{f: I \rightarrow \mathbb{C} \mid f \in D_{A}(I) ; \omega^{-1} \tau(f) \in L^{2}(I: \omega)\right\}, \\
T_{\text {max }} f=\omega^{-1} \tau(f),\left(f \in D\left(T_{\text {max }}\right)\right) ; \\
D\left(T_{\text {min }}\right)=\left\{f \mid f \in D\left(T_{\text {max }}\right) ;[f, g]_{A}(b)-[f, g]_{A}(a)=0\left(g \in D\left(T_{\text {max }}\right)\right)\right\}, \\
T_{\text {min }} f=\omega^{-1} \tau(f),\left(f \in D\left(T_{\text {min }}\right)\right),
\end{gathered}
$$

where $[f, g]_{A}(b)$ and $[f, g]_{A}(a)$ are the limits (which necessarily exist) of the bilinear forms from (2), that is $\lim _{\beta \rightarrow b}[f, g]_{A}(\beta)=[f, g]_{A}(b)$ and $\lim _{\alpha \rightarrow a}[f, g]_{A}(\alpha)=$ $[f, g]_{A}(a)$.

The following general theorem is known for the operators $T_{\max }$ and $T_{\min }$ :

TheOREM 1.2. For the operators $T_{\max }$ and $T_{\min }$ and their domains the following facts are valid:

(a) $D\left(T_{\min }\right) \subseteq D\left(T_{\max }\right)$. Domains $D\left(T_{\min }\right)$ and $D\left(T_{\max }\right)$ are dense in $L^{2}(I: \omega)$;

(b) The operator $T_{\min }$ is closed and symmetric, the operator $T_{\max }$ is closed in 
$L^{2}(I: \omega)$

(c) $\quad T_{\min }^{*}=T_{\max }$ and $T_{\max }^{*}=T_{\min }$.

All self-adjoint extensions of $T_{\min }$ appear to be the contractions of $T_{\max }$.

Let $\Omega$ be a finite or a countable set of indices. On $\Omega$, we have an Everitt-MarkusZettl multi-interval quasi-differential system $\left\{I_{i}, \tau_{i} ; \omega_{i}\right\}_{i \in \Omega}$. This EMZ system generates a family of the weighted Hilbert spaces $\left\{L^{2}\left(I_{i}: \omega_{i}\right)=L_{i}^{2}\right\}_{i \in \Omega}$ and families of minimal $\left\{T_{\min , i}\right\}_{i \in \Omega}$ and maximal $\left\{T_{\max , i}\right\}_{i \in \Omega}$ operators. Consider a respective family $\left\{T_{i}\right\}_{i \in \Omega}$ of self-adjoint extensions.

We introduce the system Hilbert space $\mathbf{L}^{2}=\oplus_{i \in \Omega} L_{i}^{2}$ consisting of vectors $\mathbf{f}=$ $\oplus_{i \in \Omega} f_{i}$, such that $f_{i} \in L_{i}^{2}$ and

$$
\|\mathbf{f}\|^{2}=\sum_{i \in \Omega}\left\|f_{i}\right\|_{i}^{2}=\sum_{i \in \Omega} \int_{I_{i}}\left|f_{i}\right|^{2} \omega_{i} d x<\infty,
$$

where $\|\cdot\|_{i}^{2}$ are the norms in $L_{i}^{2}$. In the space $\mathbf{L}^{2}$ consider the operator $T: D(T) \subseteq$ $\mathbf{L}^{2} \rightarrow \mathbf{L}^{2}$, defined on the domain

$$
D(T)=\left\{\mathbf{f} \in \oplus_{i \in \Omega} D\left(T_{i}\right) \subseteq \mathbf{L}^{2}: \sum_{i \in \Omega}\left\|T_{i} f_{i}\right\|_{i}^{2}<\infty\right\}
$$

by $T \mathbf{f}=\oplus_{i \in \Omega} T_{i} f_{i}$.

Definition 1.3. The operator $T=\oplus_{i \in \Omega} T_{i}$ is called a differential vector-operator generated by the self-adjoint extensions $T_{i}$ on an $E M Z$ system, or simply a vectoroperator. If $\Omega$ is infinite, the vector-operator $T$ is called infinite. The operators $T_{i}$ are called coordinate operators. For $\Omega^{\prime} \subset \Omega$, the operator $\oplus_{k \in \Omega^{\prime}} T_{k}$ is called a sub-vector-operator of the vector-operator $\oplus_{i \in \Omega} T_{i}$.

The following abstract preliminaries may be found, for instance, in books [17, 18].

Fix $i \in \Omega$. For each $T_{i}$ there exists a unique resolution of the identity $E_{\lambda}^{i}$ and a unitary operator $U_{i}$, making the isometrically isomorphic mapping of the Hilbert space $L_{i}^{2}$ onto the space $L^{2}\left(M_{i}, \mu_{i}\right)$, where the operator $T_{i}$ is represented as a multiplication operator. Below, we remind the structure of the mapping $U_{i}$.

We call $\phi \in L_{i}^{2}$ a cyclic vector if for each $z \in L_{i}^{2}$ there exists a Borel function $f$, such that $z=f\left(T_{i}\right) \phi$. Generally, there is no a cyclic vector in $L_{i}^{2}$ but there is a collection $\left\{\phi^{k}\right\}$ of them in $L_{i}^{2}$, such that $L_{i}^{2}=\oplus^{k} L_{i}^{2}\left(\phi^{k}\right)$, where $L_{i}^{2}\left(\phi^{k}\right)$ are $T_{i}$-invariant subspaces in $L_{i}^{2}$ generated by the cyclic vectors $\phi^{k}$. That is

$$
L_{i}^{2}\left(\phi^{k}\right)=\overline{\left\{f\left(T_{i}\right) \phi^{k}\right\}},
$$

for a varying Borel function $f$, such that $\phi^{k} \in D\left(f\left(T_{i}\right)\right)$. There exist unitary operators

$$
U^{k}: L_{i}^{2}\left(\phi^{k}\right) \rightarrow L^{2}\left(\mathbb{R}, \mu^{k}\right)
$$

where $\mu^{k}(\Delta)=\left\|E^{i}(\Delta) \phi^{k}\right\|_{i}^{2}$ for any Borel set $\Delta$. In $L^{2}\left(\mathbb{R}, \mu^{k}\right)$, the operator $T_{i}$ has the form of multiplication by $\lambda$, i.e.

$$
\left(\left.U^{k} T_{i}\right|_{L_{i}^{2}\left(\phi^{k}\right)} U^{k-1} z\right)(\lambda)=\lambda z(\lambda) .
$$

Then the operator

$$
U_{i}=\oplus^{k} U^{k}: \oplus^{k} L_{i}^{2}\left(\phi^{k}\right) \rightarrow \oplus^{k} L^{2}\left(\mathbb{R}, \mu^{k}\right)
$$


makes the spectral representation of the space $L_{i}^{2}$ onto the space $L^{2}\left(M_{i}, \mu_{i}\right)$, where $M_{i}$ is a union of nonintersecting copies of the real line (a sliced union) and $\mu_{i}=\sum_{k} \mu^{k}$. That is $\left(U_{i} T_{i} U_{i}^{-1} z\right)(\lambda)=f(\lambda) z(\lambda)$, where $z \in U\left[D\left(T_{i}\right)\right]$ and $f$ is a Borel function defined almost everywhere according to the measure $\mu_{i}$.

A vector $\phi \in L_{i}^{2}$ is called maximal relative to the operator $T_{i}$, if each measure $\left(E^{i}(\cdot) x, x\right)_{i}, x \in L_{i}^{2}$, is absolutely continuous relative to the measure $\left(E^{i}(\cdot) \phi, \phi\right)_{i}$.

For each Hilbert space $L_{i}^{2}$, there exist a unique (up to unitary equivalence) decomposition $L_{i}^{2}=\oplus_{k} L_{i}^{2}\left(\varphi_{i}^{k}\right)$, where $\varphi_{i}^{1}$ is maximal in $L_{i}^{2}$ relative to $T_{i}$, and a decreasing set of multiplicity sets $e_{k}^{i}$, where $e_{1}^{i}$ is the whole line, such that $\oplus_{k} L_{i}^{2}\left(\varphi_{i}^{k}\right)$ is equivalent with $\oplus_{k} L^{2}\left(e_{k}^{i}, \mu_{i}\right)$, where the measure of the ordered representation is defined as $\mu_{i}(\cdot)=\left(E^{i}(\cdot) \varphi_{i}^{1}, \varphi_{i}^{1}\right)_{i}$. A spectral representation of $T_{i}$ in $\oplus_{k} L^{2}\left(e_{k}^{i}, \mu_{i}\right)$ is called the ordered representation and it is unique, up to a unitary equivalence. Two operators are called equivalent, if they create the same ordered representation of their spaces.

2. The spectral representation for the vector-operator $T$. In this section we show, how the common spectral representation of the vector-operator $T$ depends on the common spectral representations of the given operators $T_{i}$. For this purpose, we first prove some auxiliary results.

Definition 2.1. For $i \in \Omega$, we introduce a sliced union of sets $M_{i}$ (see also preliminaries) as a set $M$, containing all $M_{i}$ on different copies of $\cup_{i \in \Omega} M_{i}$. The sets $M_{i}$ do not intersect in $M$, but they can superpose, i.e. two sets $M_{i}$ and $M_{j}$ superpose, if their projections in the set $\cup_{i \in \Omega} M_{i}$ intersect.

Separate arguments show, that the following auxiliary proposition is true.

Proposition 2.2. Let us have a set of measures $\mu_{i}, i \in \Omega$, defined on nonintersecting supports. If

$$
\sum_{i \in \Omega} \int_{-\infty}^{\infty} f(\lambda) d \mu_{i}(\lambda)<\infty
$$

for any Borel function $f(\lambda)$, then the following equality is true:

$$
\sum_{i \in \Omega} \int_{-\infty}^{\infty} f(\lambda) d \mu_{i}(\lambda)=\int_{-\infty}^{\infty} f(\lambda) d \sum_{i \in \Omega} \mu_{i}(\lambda) .
$$

Lemma 2.3. The identity resolution $\left\{E_{\lambda}\right\}$ of the vector-operator $T$ equals to the direct sum of the coordinate identity resolutions $\left\{E_{\lambda}^{i}\right\}$, that is:

$$
\left\{E_{\lambda}\right\}=\oplus_{i \in \Omega}\left\{E_{\lambda}^{i}\right\}
$$

Proof. A vector $\mathbf{x}$ belongs to $D(T)$ if and only if

$$
\|T \mathbf{x}\|^{2}=\sum_{i \in \Omega}\left\|T_{i} x_{i}\right\|_{i}^{2}=\sum_{i \in \Omega} \int_{-\infty}^{\infty} \lambda^{2} d\left\|E_{\lambda}^{i} x_{i}\right\|_{i}^{2}<\infty .
$$

Then, using Proposition 2.2 we find out that:

$$
\sum_{i \in \Omega} \int_{-\infty}^{\infty} \lambda^{2} d\left\|E_{\lambda}^{i} x_{i}\right\|_{i}^{2}=\int_{-\infty}^{\infty} \lambda^{2} d \sum_{i \in \Omega}\left\|E_{\lambda}^{i} x_{i}\right\|_{i}^{2} .
$$


This means, that $\mathbf{x} \in D(T)$, if and only if

$$
\int_{-\infty}^{\infty} \lambda^{2} d \sum_{i \in \Omega}\left\|E_{\lambda}^{i} x_{i}\right\|_{i}^{2}<\infty
$$

and

$$
\|T \mathbf{x}\|^{2}=\int_{-\infty}^{\infty} \lambda^{2} d \sum_{i \in \Omega}\left\|E_{\lambda}^{i} x_{i}\right\|_{i}^{2}
$$

Using the uniqueness property of an identity resolution, the last two equations show that $\oplus_{i \in \Omega}\left\{E_{\lambda}^{i}\right\}$ is the identity resolution of the vector-operator $T$. That is, according to our notations $\left\{E_{\lambda}\right\}=\oplus_{i \in \Omega}\left\{E_{\lambda}^{i}\right\}$. The lemma is proved.

Lemma 2.4. For any Borel function $f$ and any vector $\mathbf{x} \in D(f(T))$, the following equality holds: $f(T) \mathbf{x}=\left[\oplus_{i \in \Omega} f\left(T_{i}\right)\right] \mathbf{x}$.

Proof. Let $\mathbf{x} \in D(f(T))$. Then, paying attention to Proposition 2.2 and Lemma 2.3 , for any $\mathbf{y} \in \mathbf{L}^{2}$, we obtain:

$$
\begin{aligned}
(f(T) \mathbf{x}, \mathbf{y})= & \int_{-\infty}^{\infty} f(\lambda) d\left(E_{\lambda} \mathbf{x}, \mathbf{y}\right)=\int_{-\infty}^{\infty} f(\lambda) d \sum_{i \in \Omega}\left(E_{\lambda}^{i} x_{i}, y_{i}\right)_{i}= \\
& =\sum_{i \in \Omega} \int_{-\infty}^{\infty} f(\lambda) d\left(E_{\lambda}^{i} x_{i}, y_{i}\right)_{i}=\sum_{i \in \Omega}\left(f\left(T_{i}\right) x_{i}, y_{i}\right)_{i}=\left(\left[\oplus_{i \in \Omega} f(T)\right] \mathbf{x}, \mathbf{y}\right) .
\end{aligned}
$$

Since $\mathbf{y}$ is arbitrary, we have $f(T) \mathbf{x}=\left[\oplus_{i \in \Omega} f\left(T_{i}\right)\right] \mathbf{x}$. This completes the proof of the lemma. place.

For $z_{i} \in L_{i}^{2}, i \in \Omega$, define $\overline{\mathbf{z}_{\mathbf{i}}}=\left\{0, \ldots, 0, z_{i}, 0, \ldots, 0\right\} \in \mathbf{L}^{2}$, where $z_{i}$ is on the $i$-th

For each $i \in \Omega$, let $\epsilon\left(T_{i}\right)$ denote the subspectrum of the operator $T_{i}$, i.e. the set where the spectral measures of $T_{i}$ are concentrated. Note, that $\overline{\epsilon\left(T_{i}\right)}=\sigma\left(T_{i}\right)$. For instance, the subspectrum of an operator having the complete system of eigenfunctions with eigenvalues being the rational numbers of $[0,1]$ equals to $\mathbb{Q} \cap[0,1]$; the subspectrum of an operator having the continuous spectrum $[0,1]$ is assumed to equal to $(0,1)$ without loss of generality.

Consider a projecting mapping $P: M \rightarrow \cup_{i \in \Omega} M_{i}$ (see Definition 2.1), such that $P\left(\epsilon\left(T_{i}\right)\right)=\epsilon\left(T_{i}\right)$.

Definition 2.5. Let $\Omega=\cup_{k=1}^{K} A_{k}, A_{k} \cap A_{s}=\emptyset$ for $k \neq s$ and

$$
\begin{aligned}
A_{k}=\left\{s \in \Omega: \forall s, l \in A_{k}, s \neq l, P\left(\epsilon\left(T_{s}\right)\right) \cap P\left(\epsilon\left(T_{l}\right)\right)=B_{s l},\right. \\
\text { where } \left.\left\|E^{t}\left(B_{s l}\right) \varphi_{t}\right\|_{t}^{2}=0 \text { for any cyclic } \varphi_{t} \in L_{t}^{2}, t=s, l\right\} .
\end{aligned}
$$

From all such divisions of $\Omega$ we choose and fix the one, which contains the minimal number of $A_{k}$. In case all the coordinate spectra $\sigma\left(T_{i}\right)$ are simple, we define the number $\Lambda=\min \{K\}$ as the spectral index of the vector-operator $T$.

THEOREM 2.6. Let each $T_{i}$ have a cyclic vector $a_{i}$ in $L_{i}^{2}$. Then the vector-operator $T$ has $\Lambda$ cyclic vectors $\left\{\mathbf{a}_{k}\right\}_{k=1}^{\Lambda}$, having the form $\mathbf{a}_{k}=\sum_{i \in A_{k}} \overline{\mathbf{a}_{\mathbf{i}}}$. 
Proof. First we consider the case of two coordinate operators. Let $s, l \in \Omega$. Then, in order to obtain one cyclic vector in $L_{s}^{2} \oplus L_{l}^{2}$ having the form $a_{s} \oplus a_{l}$, for any $\mathbf{x}=x_{s} \oplus x_{l} \in L_{s}^{2} \oplus L_{l}^{2}$ we have to find a Borel function $f$, such that

$$
\mathbf{x}=f\left(T_{s} \oplus T_{l}\right)\left[a_{s} \oplus a_{l}\right]
$$

From Lemma 2.4 it follows that

$$
\mathbf{x}=\left[f\left(T_{s}\right) \oplus f\left(T_{l}\right)\right]\left[a_{s} \oplus a_{l}\right] .
$$

On the other hand we must obtain each space $L_{p}^{2}(p=s, l)$ by closing the set $\left\{f_{p}\left(T_{p}\right) a_{p}\right\}$, letting $f_{p}$ vary over all the Borel functions such that $a_{p} \in D\left(f_{p}\left(T_{p}\right)\right)$. If $s, l \in A_{k}$, then supposing that $f=f_{p}$ on $P\left(\epsilon\left(T_{p}\right)\right)$, we obtain the required function $f$, since any functions in the isomorphic space $L^{2}$ are considered equal on the set of measure zero. Hence, it is clear that for all $i \in A_{k}$, we may build a single cyclic vector of the form

$$
\mathbf{a}_{k}=\oplus_{i \in A_{k}} a_{i}=\sum_{i \in A_{k}} \overline{\mathbf{a}_{\mathbf{i}}}
$$

using the process described above, each time operating with a pair of operators.

We recall, that we have the minimal number of $A_{k}$. Consider the Hilbert space

$$
\left[\oplus_{i \in A_{k}} L_{i}^{2}\right] \oplus\left[\oplus_{j \in A_{q}} L_{j}^{2}\right], k \neq q .
$$

We know, that then

$$
\left[\cup_{i \in A_{k}} P\left(\epsilon\left(T_{i}\right)\right)\right] \cap\left[\cup_{j \in A_{q}} P\left(\epsilon\left(T_{j}\right)\right)\right]=B_{k q}
$$

has a non-zero spectral measure. From the reasonings described in the beginning of this proof we see, that for joining the cyclic vectors $\mathbf{a}_{k}=\oplus_{i \in A_{k}} a_{i}$ and $\mathbf{a}_{q}=\oplus_{j \in A_{q}} a_{j}$ into the one

$$
\mathbf{a}_{k}+\mathbf{a}_{q}=\sum_{i \in A_{k}} \overline{\mathbf{a}_{\mathbf{i}}}+\sum_{j \in A_{q}} \overline{\mathbf{a}_{\mathbf{j}}},
$$

we would have to derive the Hilbert space (3) by closing the set

$$
\left\{f_{k}\left(\oplus_{i \in A_{k}} T_{i}\right) \mathbf{a}_{k}\right\} \oplus\left\{f_{q}\left(\oplus_{j \in A_{q}} T_{j}\right) \mathbf{a}_{q}\right\}
$$

with varying the Borel functions $f_{k}$ and $f_{q}$, which coincide on $B_{k q}$. This is not possible, since the set of such functions is not dense in the isomorphic space $L^{2}$ (the isomorphism is understood as in the spectral representation of the space (3)). Hence, we have obtained $\Lambda$ cyclic vectors

$$
\mathbf{a}_{k}=\sum_{i \in A_{k}} \overline{\mathbf{a}_{\mathbf{i}}} \in \mathbf{L}^{2}, k=\overline{1, \Lambda}
$$

and have proved the theorem.

Corollary 2.7. Let each $T_{i}$ have a single cyclic vector. Then 1. $\Lambda=1$ if and only if the coordinate operators $T_{i}, i \in \Omega$, have almost everywhere (relatively to the spectral measure) pairwise non-superposing subspectra.

2. a) $\operatorname{card}(\Omega)<\aleph_{0}$. $\Lambda=\operatorname{card}(\Omega)$, if and only if all the coordinate operators $T_{i}$ 
have pairwise superposing subspectra; b) $\operatorname{card}(\Omega)=\aleph_{0} . \Lambda=\infty$, if and only if $T$ has an infinite sub-vector-operator, the coordinate operators of which have pairwise superposing subspectra. 2.6 .

Proof. The proof directly follows from the reasonings of the proof of Theorem

In the next section we will rigorously show what the spectral multiplicity of a vector-operator is. Nevertheless, this notation is intuitively clear. Running ahead, let us present here an example, which will show the difference between the spectral index and the spectral multiplicity of the vector-operator $T$.

EXAMPLE 1. We have a three-interval EMZ system $\left\{I_{i}, \tau_{i}\right\}_{i=1}^{3}$ (a kinetic energy, a mirror kinetic energy, an impulse):

$$
\begin{gathered}
I_{1}=[0,+\infty), \quad \tau_{1}=-\left(\frac{d}{d t}\right)^{2}, \\
D\left(T_{1}\right)=\left\{f \in D\left(T_{\max , 1}\right): f(0)+k f^{\prime}(0)=0,-\infty<k \leqslant \infty\right\} ; \\
I_{2}=[0,+\infty), \quad \tau_{2}=\left(\frac{d}{d t}\right)^{2}, \\
D\left(T_{2}\right)=\left\{f \in D\left(T_{\max , 2}\right): f(0)+s f^{\prime}(0)=0,-\infty<s \leqslant \infty\right\} ; \\
I_{3}=[0,1], \quad \tau_{3}=\frac{1}{i} \frac{d}{d t}, \quad D\left(T_{3}\right)=\left\{f \in D\left(T_{\max , 3}\right): f(0)=e^{i \alpha} f(1), \alpha \in[0,2 \pi]\right\} .
\end{gathered}
$$

If $k, s \in(-\infty, 0] \cup\{+\infty\}$ then

$$
\epsilon\left(T_{1}\right)=(0,+\infty), \epsilon\left(T_{2}\right)=(-\infty, 0), \epsilon\left(T_{3}\right)=\bigcup_{n=-\infty}^{\infty}(2 \pi n-\alpha) .
$$

For this system we have: $\{1,2,3\}=\cup_{k=1}^{2} A_{k}$ and $A_{1}=\{1,2\}, A_{2}=\{3\}$. Thus, here the spectral index does not coincide with the spectral multiplicity (which equals to 1) and equals to 2 .

The case $0<k, s<+\infty$ leads to the following

$$
\epsilon\left(T_{1}\right)=\left\{-\frac{1}{k^{2}}\right\} \cup(0,+\infty), \epsilon\left(T_{2}\right)=(-\infty, 0) \cup\left\{\frac{1}{s^{2}}\right\}, \epsilon\left(T_{3}\right)=\bigcup_{n=-\infty}^{\infty}(2 \pi n-\alpha) .
$$

If

$$
\alpha \notin\left[\bigcup_{n=-\infty}^{\infty}\left(2 \pi n+\frac{1}{k^{2}}\right)\right] \bigcup\left[\bigcup_{n=-\infty}^{\infty}\left(2 \pi n-\frac{1}{s^{2}}\right)\right]
$$

we have $A_{1}=\{1\}, A_{2}=\{2\}, A_{3}=\{3\}$. That is $\Lambda=3$ but $\oplus_{i=1}^{3} T_{i}$ has a simple spectrum.

EXAMPLE 2. Let us have a vector-operator $\oplus_{i=1}^{3} T_{i}$ with

$$
\epsilon\left(T_{1}\right)=\bigcup_{n \in \mathbb{Z}, n \geqslant 0} \pi n, \epsilon\left(T_{2}\right)=\bigcup_{n \in \mathbb{Z}, n \leqslant 0} \pi n, \epsilon\left(T_{3}\right)=\bigcup_{n \in \mathbb{Z}, n \neq 0} \pi n .
$$


Spectral index equals to 3 but spectral multiplicity equals to 2 .

Definition 2.8. A vector-operator $T=\oplus_{i \in \Omega} T_{i}$ with simple coordinate spectra $\sigma\left(T_{i}\right)$ is called distorted if its spectral index does not equal its spectral multiplicity.

Note that in the above example 1 , it is possible to unite the cyclic vectors into one just taking their direct sum (as it is shown in the proof of Theorem 2.6). But nevertheless, it is convenient to consider such operators as distorted satisfying Definition 2.8. The distorted vector-operator from Example 2 is 'completely' distorted and it is not possible to unite the coordinate cyclic vectors into a cyclic direct sum.

With some loss of technical value but more clearly for applications, Theorem 2.6 may be reformulated as

Corollary 2.9. Let each $T_{i}$ have a simple spectrum. Then undistorted vectoroperator $T$ has $\Lambda$-multiple spectrum.

Let us pass to the general case when each operator $T_{i}$ has $m_{i}$ cyclic vectors. There exists a decomposition

$$
T=\oplus_{i \in \Omega} T_{i}=\oplus_{i \in \Omega} \oplus_{k=i}^{m_{i}} T_{i}^{k}=\oplus_{s} T_{s}
$$

where each $T_{s}$ has a single cyclic vector. For the vector-operator $T$ decomposed as above, we apply Theorem 2.6 and find the spectral index $\Lambda$. It is clear, that in this case for the spectral index there exists the estimate

$$
\Lambda \geqslant \max \left\{m_{i}\right\}
$$

As it has been stated in the preliminaries, for each operator $T_{i}$ there exists the unitary operator $U_{i}$, such that $U_{i}: L_{i}^{2} \rightarrow L^{2}\left(M_{i}, \mu_{i}\right)$. Hence

$$
\oplus_{i \in \Omega} U_{i}: \oplus_{i \in \Omega} L_{i}^{2} \rightarrow \oplus_{i \in \Omega} L^{2}\left(M_{i}, \mu_{i}\right)
$$

Or, in the general case (i.e. when there are $T_{i}$ with more then one cyclic vector),

$$
\oplus_{i \in \Omega} U_{i}: \oplus_{i \in \Omega} \oplus_{k=1}^{m_{i}} L_{i, k}^{2} \rightarrow \oplus_{i \in \Omega} \oplus_{k=1}^{m_{i}} L^{2}\left(\mathbb{R}, \mu_{i}^{k}\right) .
$$

From Theorem 2.6 it follows that there exists a unitary operator

$$
V: \oplus_{i \in \Omega} \oplus_{k=1}^{m_{i}} L^{2}\left(\mathbb{R}, \mu_{i}^{k}\right)=\oplus_{s} L^{2}\left(\mathbb{R}, \mu_{s}\right) \rightarrow \oplus_{q=1}^{\Lambda} L^{2}\left(\mathbb{R}, \sum_{j \in A_{q}} \mu_{j}\right)
$$

This means that for any vector-operator $T$ there exists the unitary operator $V \oplus_{i \in \Omega} U_{i}$, which represents the space $\mathbf{L}^{2}$ on the space $L_{2}(N, \mu)$ :

$$
V \oplus_{i \in \Omega} U_{i}: \mathbf{L}^{2} \rightarrow L^{2}(N, \mu)
$$

where $N$ is the sliced union of $\Lambda$ copies of $\mathbb{R}$ and

$$
\mu=\sum_{q=1}^{\Lambda} \sum_{j \in A_{q}} \mu_{j}
$$

according to the symbols in (5). We finally obtain 
THEOREM 2.10. Let the vector-operator $T=\oplus_{i \in \Omega} T_{i}$ be undistorted and let the unitary operator $V$ be defined as in (5). If unitary operators $U_{i}$ give spectral representations of the Hilbert spaces $L_{i}^{2}$ on the spaces $L^{2}\left(M_{i}, \mu_{i}\right)$, then the unitary operator

$$
W=V \oplus_{i \in \Omega} U_{i}
$$

gives a spectral representation of the space $\mathbf{L}^{2}$ on the space $L^{2}(N, \mu)$.

Directly from the definition of a distorted vector-operator, it follows that only for undistorted vector-operators, the transform $V$ does reduce the quantity of cyclic vectors to the minimal possible. Note, that distorted differential vector-operators appear to be frequent objects if vector-operators are considered on a set of closed bounded intervals, and on the other hand quite rare, if coordinate operators have continuous spectra. For them Theorem 2.10 is not efficient and needs to be strengthened. Such strengthening is the construction of an ordered representation for arbitrary (distorted or not) differential vector-operators, the process which seems to be essential for further development of spectral theory of vector-operators.

\section{The ordered spectral representation for the vector-operator $T$.}

THEOREM 3.1. If $\theta_{i}$ and $\left\{e_{n}^{i}\right\}_{n=1}^{m_{i}}$ are measures and multiplicity sets of ordered representations for coordinate operators $T_{i}, i \in \Omega$, then there exist processes $\operatorname{Pr}_{1}$ and $\mathrm{Pr}_{2}$, such that the measure

$$
\theta=\operatorname{Pr}_{1}\left(\left\{\theta_{i}\right\}_{i \in \Omega}\right)
$$

is the measure of an ordered representation and the sets

$$
s_{n}=\operatorname{Pr}_{2}\left(\left\{e_{k}^{i}\right\}_{i \in \Omega ; k=\overline{1, m_{i}}}\right)
$$

are the canonical multiplicity sets of the ordered representation of the operator $T$. Thus, the unitary representation of the space $\mathbf{L}^{2}$ on the space $\oplus_{n} L^{2}\left(s_{n}, \theta\right)$ is the ordered representation and it is unique up to unitary equivalence.

Proof. We divide the proof into units for convenience. Units (A) and (B) represent the process, which we call 'the process of division on subspectra'.

(A) Let $a_{i}$ be maximal vectors relative to the operators $T_{i}$ in $L_{i}^{2}$. We want to find a maximal vector relative to the vector-operator $T$. We know, that the vector $\oplus_{i \in \Omega} a_{i}$ does not give a single measure, if a set $P\left(\epsilon\left(T_{i}\right)\right) \cap P\left(\epsilon\left(T_{j}\right)\right)$ has a non-zero spectral measure for $i \neq j$. Consider restrictions $\left.T_{i}\right|_{L_{i}^{2}\left(a_{i}\right)}=T_{i}^{\prime}$. Since all the operators $T_{i}^{\prime}$ have single cyclic vectors $a_{i}$, we can divide $\Omega$ into $A_{k}, k=\overline{1, \Lambda}$ (see Definition 2.5) and apply Theorem 2.6 for the operator $\oplus_{i \in \Omega} T_{i}^{\prime}$. Thus, we have derived $\Lambda$ vectors $\mathbf{a}^{k}=\oplus_{j \in A_{k}} a_{j}$, which are maximal in the respective spaces $\mathbf{L}^{2}\left(\mathbf{a}^{k}\right)=\oplus_{j \in A_{k}} L_{j}^{2}\left(a_{j}\right)$. Indeed, this is obvious for the case $\operatorname{card}\left(A_{k}\right)<\aleph_{0}$. For the infinite case, if arbitrary $\mathbf{y}=\oplus_{j \in A_{k}} y_{j} \in \mathbf{L}^{2}\left(\mathbf{a}^{k}\right)$ and if

$$
\left(\left[\oplus_{j \in A_{k}} E^{j}\right](\cdot) \mathbf{a}^{k}, \mathbf{a}^{k}\right)=\sum_{j \in A_{k}}\left(E^{j}(\cdot) a_{j}, a_{j}\right)_{j}=0
$$

then from the maximality of the vectors $a_{j}$ for all $j \in A_{k}$, and since $P\left(\epsilon\left(T_{j}^{\prime}\right)\right) \cap P\left(\epsilon\left(T_{k}^{\prime}\right)\right)$ has zero spectral measures for $j \neq k$, we obtain

$$
\sum_{j \in A_{k}}\left(E^{j}(\cdot) y_{j}, y_{j}\right)_{j}=\left(\left[\oplus_{j \in A_{k}} E^{j}\right](\cdot) \mathbf{y}, \mathbf{y}\right)=0
$$


which follows from the convergence to zero of the series with the positive maximal elements (6). Thus, in particular, we have constructed a maximal vector in $\mathbf{L}^{2}$ for the case $\Lambda=1$.

(B) Let now $1<\Lambda<\infty$. Define $T^{k}=\oplus_{j \in A_{k}} T_{j}^{\prime}$. For any two operators $T^{k}$ and $T^{s}, k \neq s$, let us introduce the sets $\epsilon_{k, s}=P\left(\epsilon\left(T^{k}\right)\right) \cap P\left(\epsilon\left(T^{s}\right)\right)$ and $\epsilon_{k}=P\left(\epsilon\left(T^{k}\right)\right) \backslash$ $\epsilon_{k, s}$. There exist unitary representations $U^{k}: \mathbf{L}^{2}\left(\mathbf{a}^{k}\right) \rightarrow L^{2}\left(\mathbb{R}, \mu_{\mathbf{a}^{k}}\right)$ (see formula (5) supposing there $\Lambda=1$ ). Consider measures $\mu_{k}$ and $\mu_{k, s}$, defined as

$$
\mu_{k, s}(e)=\mu_{\mathbf{a}^{k}}\left(e \cap \epsilon_{k, s}\right) \text { and } \mu_{k}(e)=\mu_{\mathbf{a}^{k}}\left(e \cap \epsilon_{k}\right),
$$

for any measurable set $e$. For any operator $T^{k}$ (with respect to $T^{s}$ ), measures $\mu_{k}$ and $\mu_{k, s}$ are mutually singular and $\mu_{k}+\mu_{k, s}=\mu_{\mathbf{a}^{k}}$; therefore

$$
L^{2}\left(\mathbb{R}, \mu_{\mathbf{a}^{k}}\right)=L^{2}\left(\mathbb{R}, \mu_{k}\right) \oplus L^{2}\left(\mathbb{R}, \mu_{k, s}\right)
$$

This means that (according to our designations):

$$
U^{k^{-1}}: L^{2}\left(\mathbb{R}, \mu_{\mathbf{a}^{k}}\right) \longrightarrow \mathbf{L}^{2}\left(\mathbf{a}_{k}^{k}\right) \oplus \mathbf{L}^{2}\left(\mathbf{a}_{k, s}^{k}\right)
$$

and $\mathbf{a}^{k}=\mathbf{a}_{k}^{k} \oplus \mathbf{a}_{k, s}^{k}$, where $\mathbf{a}_{k}^{k}$ and $\mathbf{a}_{k, s}^{k}$ form the measures $\mu_{k}$ and $\mu_{k, s}$ respectively. Define also as $\max \{w, \psi\}$ the vector, which is maximal of the two vectors in the brackets (Note, that this designation is valid only for vectors, considered on the same set. In order not to complicate the investigation we assume here that any two vectors are comparable in this sense. In order to achieve this, it is enough to decompose each coordinate operator $T_{i}$ into the direct sum $T_{i}^{p p} \oplus T_{i}^{c o n t}$, where the operators have respectively pure point and continuous spectra. Then after redesignation we obtain the equivalent vector-operator to the initial vector-operator $\oplus T_{i}$ ).

Consider first two operators $T^{1}$ and $T^{2}$. It is clear, that the vector

$$
\mathbf{a}^{1 \oplus 2}=\mathbf{a}_{1}^{1} \oplus \mathbf{a}_{2}^{2} \oplus \max \left\{\mathbf{a}_{1,2}^{1}, \mathbf{a}_{2,1}^{2}\right\}
$$

is maximal in $\mathbf{L}^{2}\left(\mathbf{a}^{1}\right) \oplus \mathbf{L}^{2}\left(\mathbf{a}^{2}\right)$. Note, that $\mathbf{a}_{1}^{1}$ and $\mathbf{a}_{2}^{2}$ and they both may equal zero. The maximal vector in $\mathbf{L}^{2}\left(\mathbf{a}^{1}\right) \oplus \mathbf{L}^{2}\left(\mathbf{a}^{2}\right) \oplus \mathbf{L}^{2}\left(\mathbf{a}^{3}\right)$ will have the form:

$$
\mathbf{a}^{1 \oplus 2 \oplus 3}=\mathbf{a}_{1 \oplus 2}^{1 \oplus 2} \oplus \mathbf{a}_{3}^{3} \oplus \max \left\{\mathbf{a}_{1 \oplus 2,3}^{1 \oplus 2}, \mathbf{a}_{3,1 \oplus 2}^{3}\right\}
$$

Continuing this process, we obtain a maximal vector in the main space $\mathbf{L}^{2}$ :

$$
\mathbf{a}^{1 \oplus \cdots \oplus \Lambda}=\mathbf{a}_{1 \oplus \cdots \oplus \Lambda-1}^{1 \oplus \cdots \oplus \Lambda-1} \oplus \mathbf{a}_{\Lambda}^{\Lambda} \oplus \max \left\{\mathbf{a}_{1 \oplus \cdots \oplus \Lambda-1, \Lambda}^{\left.1 \oplus \cdots \oplus \mathbf{a}_{\Lambda, 1 \oplus \cdots \oplus \Lambda-1}^{\Lambda}\right\}}\right.
$$

Formula 7 may be simplified, if we divide the measures $\mu_{\mathbf{a}^{k}}$ into continuous and pure point components, that is $\mu_{\mathbf{a}^{k}}=\mu_{\mathbf{a}^{k}}^{\text {cont }}+\mu_{\mathbf{a}^{k}}^{p p}$. Then $\mathbf{a}^{k}=\mathbf{a}^{k, \text { cont }} \oplus \mathbf{a}^{k, p p}$. Relatively to any operator $T^{s}, k \neq s$, we have

$$
\mathbf{a}^{k, c o n t}=\mathbf{a}_{k}^{k, \text { cont }} \oplus \mathbf{a}_{k, s}^{k, c o n t} \text { and } \mathbf{a}^{k, p p}=\mathbf{a}_{k}^{k, p p} \oplus \mathbf{a}_{k, s}^{k, p p} .
$$

Now we can repeat the process described above in (B), separately for the continuous and the pure point parts. Since measures with the same null set may be considered equivalent, we have

$$
\max \left\{w^{c o n t}, \psi^{\text {cont }}\right\}=\text { either } w^{\text {cont }} \text { or } \psi^{\text {cont }}
$$




$$
\max \left\{w^{p p}, \psi^{p p}\right\}=\operatorname{either} w^{p p} \text { or } \psi^{p p},
$$

for any two vectors $w$ and $\psi$. Thus we obtain

$$
\mathbf{a}^{1 \oplus \cdots \oplus \Lambda, \text { cont }}=\mathbf{a}^{1, \text { cont }} \oplus\left[\oplus_{j=2}^{\Lambda} \mathbf{a}_{j}^{j, \text { cont }}\right] .
$$

Similarly,

$$
\mathbf{a}^{1 \oplus \cdots \oplus \Lambda, p p}=\mathbf{a}^{1, p p} \oplus\left[\oplus_{j=2}^{\Lambda} \mathbf{a}_{j}^{j, p p}\right]
$$

Since $\max \left\{w^{\text {cont }}, \psi^{p p}\right\}=\psi^{p p}$, we finally derive

$$
\mathbf{a}^{1 \oplus \cdots \oplus \Lambda}=\mathbf{a}^{1 \oplus \cdots \oplus \Lambda, p p} \oplus \mathbf{a}_{1 \oplus \cdots \oplus \Lambda}^{1 \oplus \cdots \oplus, \text { cont }} .
$$

Let $\Lambda=\infty$. We obtain $\mathbf{a}^{1 \oplus \cdots \oplus \Lambda}$ as a vector which satisfies the following equality:

$$
\left\|\left[\oplus_{i \in \Omega} E^{i}(\cdot)\right] \mathbf{a}^{1 \oplus \cdots \oplus \Lambda}\right\|^{2}=\lim _{L \rightarrow \infty}\left\|\left[\oplus_{j=1}^{L} E^{j}(\cdot)\right] \mathbf{a}^{1 \oplus \cdots \oplus L}\right\|^{2}
$$

since the limit on the right side exists. Indeed $\lim _{L \rightarrow \infty}\left\|\left[\oplus_{j=1}^{L} E^{j}(\cdot)\right] \mathbf{a}^{1 \oplus \cdots \oplus L}\right\|^{2}$ can be rewritten as $\sum_{j=1}^{\infty}\left\|E^{j}(\cdot) \widehat{a_{j}}\right\|_{j}^{2}$, where $\widehat{a_{j}}$ are the restricted $a_{j}$. Noticing that

$$
\sum_{j=1}^{\infty}\left\|E^{j}(\cdot) \widehat{a_{j}}\right\|_{j}^{2} \leqslant \sum_{j=1}^{\infty}\left\|E^{i}(\cdot) a_{j}\right\|_{j}^{2}<\infty
$$

we prove the convergence (without loss of generality, the vectors $a_{i}$ can be always chosen such, that $\left.\sum_{i=1}^{\infty}\left\|a_{i}\right\|_{i}^{2}<\infty\right)$.

(C) The next step is to build the measure of the ordered representation for the vector-operator. From Lemma 2.3 and the reasonings above, it follows that such a measure will be

$$
\theta(\cdot)=\left(\left[\oplus_{i \in \Omega} E^{i}(\cdot)\right] \mathbf{a}^{1 \oplus \cdots \oplus \Lambda}, \mathbf{a}^{1 \oplus \cdots \oplus \Lambda}\right) .
$$

Thus we have constructed the process $P r_{1}$.

(D) The final step is to construct the canonical multiplicity sets $s_{n}$ of the vectoroperator; $s_{1}$ is the whole line; $s_{2}$ must contain all the spectrum the multiplicity of which exceeds or equals to 2 . For this purpose, we are primarily to unite all $e_{2}^{i}$. But, nevertheless, $\cup_{i} e_{2}^{i}$ will not include all the sets of multiplicity $\geqslant 2$, since we know that if $P\left(e_{1}^{i} \backslash e_{2}^{i}\right) \cap P\left(e_{1}^{j} \backslash e_{2}^{j}\right)$ has a non-zero spectral measure, all the intersections of this sort will represent the multiplicity 2 and should be included into $s_{2}$ (since then it is not possible to construct a single cyclic vector). That is $s_{2}=\left(\cup_{i} P\left(e_{2}^{i}\right)\right) \cup\left(\cup \cap\left(P\left(e_{1}^{i} \backslash e_{2}^{i}\right)\right)\right.$. Using this idea and the fact that an infinite intersection of measurable sets is a measurable set, by induction we may finally build $s_{n}$ :

$$
s_{n}=\left[\bigcup_{i} P\left(e_{n}^{i}\right)\right] \bigcup\left[\bigcup_{\sum m_{i} \geqslant n} \bigcap P\left(e_{m_{i}}^{i} \backslash e_{m_{i}+1}^{i}\right)\right]
$$

We have constructed the process $\mathrm{Pr}_{2}$. 
The constructed measure and the multiplicity sets induce the ordered representation. It is known that such a representation is unique up to unitary equivalence.

Let us return to the example 2. For the distorted vector-operator $T_{1} \oplus T_{2} \oplus T_{3}$, two spectral measures will be constructed on vectors $\mathbf{a}^{1 \oplus 2 \oplus 3}$ and

$$
\min \left\{a_{1,2}^{1}, a_{2,1}^{2}\right\} \oplus \min \left\{a_{2,3}^{2}, a_{3,2}^{3}\right\} \oplus \min \left\{a_{3,1}^{3}, a_{1,3}^{1}\right\},
$$

where the sense of the minimums is clear.

Here the term 'distorted vector-operator' is clearly explained by the form of its cyclic vectors. The multiplicity set $e_{2}$ will be

$$
\left[P\left(\epsilon\left(T_{1}\right)\right) \cap P\left(\epsilon\left(T_{2}\right)\right)\right] \cup\left[P\left(\epsilon\left(T_{1}\right)\right) \cap P\left(\epsilon\left(T_{3}\right)\right)\right] \cup\left[P\left(\epsilon\left(T_{2}\right)\right) \cap P\left(\epsilon\left(T_{3}\right)\right)\right] .
$$

Using the obtained spectral representation we can construct equivalence classes in families of self-adjoint operators:

Definition 3.2. Two families of self-adjoint extensions $\left\{T_{i}\right\}_{i=1}^{N}$ and $\left\{S_{j}\right\}_{j=1}^{L}$ are called equivalent, if respective vector-operators $\oplus_{i=1}^{N} T_{i}$ and $\oplus_{j=1}^{L} S_{j}$ are equivalent.

Note, that if two families $\left\{T_{i}\right\}_{i=1}^{N}$ and $\left\{S_{j}\right\}_{j=1}^{L}$ are equivalent, it is not necessarily the case that $N=L$ and $T_{i}$ is equivalent with $S_{i}$.

4. The structure of the ordered spectral representation. Up to now, we have not used the structure of the coordinate operators as differential operators. In this section we make precise the ordered representation obtained in the previous section.

Let $I=\bigvee_{i \in \Omega} I_{i}$ denote the sliced union of intervals $I_{i}$. Similarly, $I^{k}=\bigvee_{j \in A_{k}} I_{j}$. If $x_{i}$ are variables on $I_{i}$, then $\vee x_{i}$ will designate a variable either on $I$ or $I^{k}$ depending on the context. This notation shows, that a vector-function

$$
z=\left\{z_{1}\left(x_{1}\right), \ldots, z_{n}\left(x_{n}\right), \ldots\right\}
$$

on $I$ or $I^{k}$ may be written as $z\left(\vee x_{i}\right)$. In particular, we may also write $\mathbf{z}\left(\vee x_{i}\right)$ instead of $\mathbf{z}=\oplus_{i \in \Omega} z_{i}$.

Let us introduce the space $\oplus_{i \in \Omega} L^{\infty}\left(I_{i}^{n}\right)$. Here, $\mathbf{z}\left(\vee x_{i}\right) \in \oplus_{i \in \Omega} L^{\infty}\left(I_{i}^{n}\right)$ means that

$$
\sup _{i \in \Omega}\left\{\operatorname{ess} \sup _{x_{i} \in I_{i}^{n}}\left|z_{i}\left(x_{i}\right) \chi_{I_{i}^{n}}\left(x_{i}\right)\right|\right\}<\infty,
$$

where for each $i$, families $\left\{I_{i}^{n}\right\}_{n=1}^{\infty}$ represent compact subintervals of $I_{i}$, such that $\cup_{n=1}^{\infty} I_{i}^{n}=I_{i}$ and $\chi$ is the characteristic function. In [4, Lemma 2.1], it was shown that $\oplus_{i \in \Omega} L^{\infty}\left(I_{i}^{n}\right)=\left(\oplus_{i \in \Omega} L^{1}\left(I_{i}^{n}\right)\right)^{*}$, where the space of Lebesgue-integrable vectorfunctions $\oplus_{i \in \Omega} L^{1}\left(I_{i}^{n}\right)$ is defined analogously to $\mathbf{L}^{2}$.

We also need to introduce a symbolic integral $\int_{\bigvee J_{i}} f\left(\vee x_{i}\right) d\left(\vee x_{i}\right)$ defined by:

$$
\int_{\bigvee J_{i}} f\left(\bigvee x_{i}\right) d\left(\bigvee x_{i}\right)=\oplus_{i} \int_{J_{i}} f_{i}\left(x_{i}\right) d x_{i},
$$

where $f\left(\vee x_{i}\right)$ is understood to be measurable relatively to $d\left(\vee x_{i}\right)$, if and only if $f_{i}\left(x_{i}\right)$ are measurable relatively to Lebesgue measures $d x_{i}$. Then

$$
\int_{\bigvee J_{i}} f\left(\vee x_{i}\right) d\left(\vee x_{i}\right)<\infty
$$


if and only if $\sup _{i} \int_{J_{i}} f_{i}\left(x_{i}\right) d x_{i}<\infty$.

The main result of the current work is to prove the following theorem:

THEOREM 4.1. Let $T$ be a self-adjoint vector-operator, generated by an EMZ system $\left\{I_{i}, \tau_{i}\right\}_{i \in \Omega}$. Let $U$ be an ordered representation of the space $\mathbf{L}^{2}=\oplus_{i \in \Omega} L^{2}\left(I_{i}\right)$ relative to $T$ with the measure $\theta$ and the multiplicity sets $s_{k}, k=\overline{1, m}$. Then there exist kernels $\Theta_{k}\left(\vee x_{i}, \lambda\right)$, measurable relative to $d\left(\vee x_{i}\right) \times \theta$, such that $\Theta_{k}\left(\vee x_{i}, \lambda\right)=0$ for $\lambda \in \mathbb{R} \backslash s_{k}$ and $\left(\oplus_{i \in \Omega} \tau_{i}-\lambda\right) \Theta_{k}\left(\vee x_{i}, \lambda\right)=0$ for each fixed $\lambda$. Moreover,

$$
\begin{gathered}
\int_{\Delta}\left|\Theta_{k}\left(\vee x_{i}, \lambda\right)\right|^{2} d \theta(\lambda) \in \oplus_{i \in \Omega} L^{\infty}\left(I_{i}^{n}\right) \forall n \in \mathbb{N} . \\
(U \mathbf{w})^{k}(\lambda)=\lim _{n \rightarrow \infty} \int_{I^{n}} \mathbf{w}\left(\vee x_{i}\right) \overline{\Theta_{k}\left(\vee x_{i}, \lambda\right)} d\left(\vee x_{i}\right), \quad \mathbf{w} \in \mathbf{L}^{2},
\end{gathered}
$$

where the limit exists in $L^{2}\left(s_{k}, \theta\right)$. The kernels $\left\{\Theta_{k}\left(\vee x_{i}, \lambda\right)\right\}_{k=1}^{n}, n \leqslant m$, are linearly independent as vector-functions of the first variable almost everywhere relatively to the measure $\theta$ on $s_{n}$.

Proof. Fix $i$. If $\theta_{i}$ and $\left\{e_{p}^{i}\right\}_{p=1}^{m_{i}}$ are respectively the measure and the multiplicity sets of an ordered representation for $T_{i}$, then there exists the decomposition $L_{i}^{2}=$ $\oplus_{p=1}^{m_{i}} L^{2}\left(e_{p}^{i}, \theta_{i}\right)$, which implies $T_{i}=\oplus_{p=1}^{m_{i}} T_{i}^{p}$ and $L^{2}\left(e_{p}^{i}, \theta_{i}\right)$ are $T_{i}^{p}$-invariant. For vector-operator $\left(\oplus_{i \in \Omega} \oplus_{p=1}^{m_{i}} T_{i}^{p}\right) \rightarrow$ redesignate $\rightarrow \oplus_{s} T_{s}, s=\{i, p\} \in \Omega_{1}$, we may write $\Omega_{1}=\cup_{k=1}^{\Lambda} A_{k}$.

Let us separate the proof into units for convenience.

(A) For each $T_{j}, j \in A_{k} \subset \Omega_{1}$ and $k=\overline{1, \Lambda}$, there exists a single cyclic vector $a_{j} \in L_{j}^{2}$ and [18, XII.3, Lemma 9 and XIII.5, Theorem $\left.1(\mathrm{I})\right]$ a function $W_{j}\left(x_{j}, \lambda\right)$ defined on $I_{j} \times e_{j}$ (note, that for a fixed $i \in \Omega, I_{j}=I_{i}$ for all $p=\overline{1, m_{i}}$ ) and measurable relatively to $d x_{j} \times \mu_{a_{j}}$, such that $W_{j}\left(x_{j}, \lambda\right)=0, \lambda \in \mathbb{R} \backslash e_{j}$ and for any bounded $\Delta \subset e_{j}$ :

$$
\int_{\Delta}\left|W_{j}\left(x_{j}, \lambda\right)\right|^{2} d \mu_{a_{j}}(\lambda) \in L^{\infty}\left(I_{j}^{n}\right), \quad n \in \mathbb{N} .
$$

Also

$$
\left(E^{j}(\Delta) F_{j}\left(T_{j}\right) a_{j}\right)\left(x_{j}\right)=\int_{\Delta} W_{j}\left(x_{j}, \lambda\right) F_{j}(\lambda) d \mu_{a_{j}}(\lambda),
$$

for any $F_{j} \in L^{2}\left(e_{j}, \mu_{a_{j}}\right)$. On $I^{k}=\bigvee_{j \in A_{k}} I_{j}$, we construct the vector-function

$$
W^{k}\left(\vee x_{j}, \lambda\right)=\left\{W_{1}\left(x_{1}, \lambda\right), \ldots, W_{n}\left(x_{n}, \lambda\right), \ldots\right\},
$$

which is obviously measurable relative to $d\left(\vee x_{j}\right) \times \sum \mu_{a_{j}}$. Since $W_{j}(\cdot, \lambda) \in L^{2}\left(\Delta, \mu_{a_{j}}\right)$, then substituting $\overline{W_{j}(\cdot, \lambda)}=\overline{W_{j}(\lambda)}$ in (12) in place of $F_{j}$, we obtain

$$
\left(E^{j}(\Delta) \overline{W_{j}\left(T_{j}\right)} a_{j}\right)\left(x_{j}\right)=\int_{\Delta}\left|W_{j}\left(x_{j}, \lambda\right)\right|^{2} d \mu_{a_{j}}(\lambda)
$$

Remembering, that $P\left(\epsilon\left(T_{s}\right)\right) \cap P\left(\epsilon\left(T_{j}\right)\right)$ has zero measure, for $s \neq j$ and $s, j \in A_{k}$, we obtain

$$
\left(\left[\oplus_{j \in A_{k}} E^{j}\right](\Delta) \overline{W^{k}\left(\oplus_{j \in A_{k}} T_{j}\right)} \mathbf{a}^{k}\right)\left(\vee x_{j}\right)=\int_{\Delta}\left|W^{k}\left(\vee x_{j}, \lambda\right)\right|^{2} d \mu_{\mathbf{a}^{k}}(\lambda),
$$


where $\mathbf{a}^{k}=\oplus_{j \in A_{k}} a_{j}$.

Since elements $f_{j}$ from $D\left(T_{j}\right)$ are continuous and thus essentially bounded on $I_{i}^{n}$ for any $n \in \mathbb{N}, \oplus_{j \in A_{k}} f_{j} \in \oplus_{j \in A_{k}} D\left(T_{j}\right)$ implies that

$$
\text { Range }\left[\oplus_{j \in A_{k}} E^{j}\right](\Delta) \subseteq \oplus_{j \in A_{k}} D\left(T_{j}\right) \subset \oplus_{j \in A_{k}} L^{\infty}\left(I_{j}^{n}\right)
$$

and hence, we obtain

$$
\int_{\Delta}\left|W^{k}\left(\vee x_{j}, \lambda\right)\right|^{2} d \mu_{\mathbf{a}^{k}} \in \oplus_{j \in A_{k}} L^{\infty}\left(I_{j}^{n}\right) \forall n \in \mathbb{N} .
$$

In $\left[18\right.$, XIII.5, Theorem 1(I)] it was shown that if we have ordered representations $U_{i}$ of $L_{i}^{2}$ relative to the operators $T_{i}, i \in \Omega$, the following formula is valid for $j \in \Omega_{1}$ :

$$
\left(U_{j} w_{j}\right)(\lambda)=\lim _{n \rightarrow \infty} \int_{I_{j}^{n}} w\left(x_{j}\right) \overline{W_{j}\left(x_{j}, \lambda\right)} d x_{j}, \quad w_{j} \in L_{j}^{2},
$$

where the limit exists in $L^{2}\left(e_{j}, \mu_{a_{j}}\right)$. Taking direct sums in both sides of the last equality, for each system of compact subintervals we obtain

$$
\left(U^{k} \oplus_{j \in A_{k}} w_{j}^{n}\right)(\lambda)=\oplus_{j \in A_{k}} \int_{I_{j}^{n}} w_{j}\left(x_{j}\right) \overline{W_{j}\left(x_{j}, \lambda\right)} d x_{j}, \quad w_{j}^{n}=w_{j} \chi_{I_{j}^{n}} .
$$

From (13), it follows that for any bounded Borel set $\Delta \in e_{j}$ and $I^{k, n}=\bigvee_{j \in A_{k}} I_{j}^{n}$,

$$
\int_{I^{k, n}} \int_{\Delta}\left|W^{k}\left(\vee x_{j}, \lambda\right)\right|^{2} d \mu_{\mathbf{a}^{k}} d\left(\vee x_{j}\right)<\infty
$$

and since $\mathbf{w}^{k}=\oplus_{j \in A_{k}} w_{j}\left(x_{j}\right)$ is assumed to belong to $\oplus_{j \in A_{k}} L_{j}^{2}$, we may write:

$$
\left(U^{k} \mathbf{w}^{k, n}\right)(\lambda)=\int_{I^{k, n}} \mathbf{w}^{k}\left(\vee x_{j}\right) \overline{W^{k}\left(\vee x_{j}, \lambda\right)} d\left(\vee x_{j}\right) .
$$

Taking the limit in the both sides and defining $\mathbf{w}^{k}=\oplus_{j} \lim _{n \rightarrow \infty} w_{j}^{n}$ we obtain the formula

$$
\left(U^{k} \mathbf{w}^{k}\right)(\lambda)=\lim _{n \rightarrow \infty} \int_{I^{k, n}} \mathbf{w}^{k}\left(\vee x_{j}\right) \overline{W^{k}\left(\vee x_{j}, \lambda\right)} d\left(\vee x_{j}\right), \quad \mathbf{w}^{k} \in \oplus_{j \in A_{k}} L_{j}^{2} .
$$

Note, that since for all $p=\overline{1, m_{i}}$ there exists the equality $\left(\tau_{i}-\lambda\right) W_{i}^{p}=0$ (see $[18$, XIII.5, Theorem 1]), it is obvious that $\left(\oplus_{j \in A_{k}} \tau_{j}-\lambda\right) W^{k}=0$, where $\tau_{j}=\tau_{i}$ for a fixed $i$ and all $p=\overline{1, m_{i}}$. If $P\left(\epsilon\left(T_{i}\right)\right) \cap P\left(\epsilon\left(T_{j}\right)\right)$ has zero spectral measures for all $i, j \in \Omega$, then $A_{k}: \Omega_{1}=\cup_{k=1}^{\Lambda_{1}} A_{k}$ may be constructed such that $A_{k}$ contains of indices $\{i, k\}, i \in \Omega, k=\overline{1, \max _{i}\left\{m_{i}\right\}}$.

(B) Consider the set of indices $\Omega_{2}=\left\{j \in \Omega_{1}: j=\{i, 1\}, i \in \Omega\right\}$. Construct $A_{k}: \Omega_{2}=\cup_{k=1}^{\Lambda_{2}} A_{k}$. Apply the reasonings used in (A), considering everywhere $\Omega_{2}$ instead of $\Omega_{1}$. Hence, for each $A_{k}$ and we find a vector-function $W_{1}^{k}\left(\vee x_{j}, \lambda\right)$ which is the solution of the equation $\left(\oplus_{j \in A_{k}} \tau_{j}-\lambda\right) \mathbf{y}=0$. Consider $W_{1}^{k}$ and $W_{1}^{s}$ for $s \neq k$. For $\mathbf{a}^{k}$ there exists the decomposition $\mathbf{a}^{k}=\mathbf{a}_{k}^{k} \oplus \mathbf{a}_{k, s}^{k}$ (see the proof of Theorem 3.1). This fact induces the decomposition for $W_{1}^{k}: W_{1}^{k}=W_{1, k}^{k} \oplus W_{1, k, s}^{k}$. It is clear that being the restrictions of $W_{1}^{k}$, the vector-functions $W_{1, k}^{k}$ and $W_{1, k, s}^{k}$ are also the solutions 
of the equation $\left(\oplus_{j \in A_{k}} \tau_{j}-\lambda\right) \mathbf{y}=0$. They, along with $\mathbf{a}_{k}^{k}$ and $\mathbf{a}_{k, s}^{k}$ define unitary transformations $U_{k}^{k}$ and $U_{k, s}^{k}$ by formula (14), such that:

$$
U_{k}^{k}: \mathbf{L}^{2}\left(\mathbf{a}_{k}^{k}\right) \rightarrow L^{2}\left(\mathbb{R}, \mu_{k}\right) \text { and } U_{k, s}^{k}: \mathbf{L}^{2}\left(\mathbf{a}_{k, s}^{k}\right) \rightarrow L^{2}\left(\mathbb{R}, \mu_{k, s}\right)
$$

(see the definitions in the proof of Theorem 3.1). This implies, that the decomposition $W^{k}=W_{1, k}^{k} \oplus W_{1, k, s}^{k}$ is correct.

Define as $\max \left\{W_{1, k, s}^{k}, W_{1, s, k}^{s}\right\}$ the vector-function, which corresponds to the vector $\max \left\{\mathbf{a}_{k, s}^{k}, \mathbf{a}_{s, k}^{s}\right\}$, respectively $\min \left\{W_{1, k, s}^{k}, W_{1, s, k}^{s}\right\}$ as the vector-function which corresponds to that $\mathbf{a}_{k, s}^{k}$ or $\mathbf{a}_{s, k}^{s}$, which is not maximal of the two.

(C) Without loss of generality, suppose that $k=1$ and $s=2$. From the reasonings presented in Unit (A) of this proof, it follows that

$$
\Theta_{1}^{1 \oplus 2}=W_{1,1}^{1} \oplus W_{1,2}^{2} \oplus \max \left\{W_{1,1,2}^{1}, W_{1,2,1}^{2}\right\}
$$

is correctly constructed vector-function satisfying the statement of the theorem for the case $T=\left[\oplus_{j \in A_{1}} T_{j}\right] \oplus\left[\oplus_{q \in A_{2}} T_{q}\right]$. Apply the above described process to $\Theta_{1}^{1 \oplus 2}$ and $W_{1}^{3}$ to obtain the correctly constructed vector-function:

$$
\Theta_{1}^{1 \oplus 2 \oplus 3}=\Theta_{1,1 \oplus 2}^{1 \oplus 2} \oplus W_{1,3}^{3} \oplus \max \left\{\Theta_{1,1 \oplus 2,3}^{1 \oplus 2}, W_{1,3,1 \oplus 2}^{3}\right\} .
$$

Continuing this process, we finally obtain:

$$
\begin{aligned}
\Theta_{1}\left(\vee x_{i}, \lambda\right) & =\Theta_{1}^{1 \oplus \cdots \oplus \Lambda_{2}}= \\
& =\Theta_{1,1 \oplus \cdots \oplus \Lambda_{2}-1}^{1 \oplus \cdots \oplus \Lambda_{2}-1} \oplus W_{1, \Lambda_{2}}^{\Lambda_{2}} \oplus \max \left\{\Theta_{1,1 \oplus \cdots \oplus \Lambda_{2}-1, \Lambda_{2}}^{1 \oplus \cdots \oplus \Lambda_{2}-1}, W_{1, \Lambda_{2}, 1 \oplus \cdots \oplus \Lambda_{2}-1}^{\Lambda_{\Lambda_{2}}}\right\},
\end{aligned}
$$

where in the case of $\Lambda_{2}=\infty, \Theta_{1}^{1 \oplus \cdots \oplus \Lambda_{2}}$ is the function which satisfies (analogously to (8)):

$$
\begin{aligned}
& \left(\left[\oplus_{i \in \Omega} E^{i}(\Delta)\right] \int_{\Delta} \Theta_{1}^{1 \oplus \cdots \oplus \Lambda_{2}} d \theta(\lambda), \int_{\Delta} \Theta_{1}^{1 \oplus \cdots \oplus \Lambda_{2}} d \theta(\lambda)\right)= \\
& =\lim _{L \rightarrow \infty}\left(\left[\oplus_{j=1}^{L} E^{j}(\Delta)\right] \int_{\Delta} \Theta_{1}^{1 \oplus \cdots \oplus L} d \theta_{L}(\lambda), \int_{\Delta} \Theta_{1}^{1 \oplus \cdots \oplus L} d \theta_{L}(\lambda)\right),
\end{aligned}
$$

for any bounded Borel set $\Delta$, where $\theta_{L}(\cdot)=\left(\left[\oplus_{j=1}^{L} E^{j}(\cdot)\right] \mathbf{a}^{1 \oplus \cdots \oplus L}, \mathbf{a}^{1 \oplus \cdots \oplus L}\right)$ is the measure of the ordered representation of the space $\oplus_{j=1}^{L} L_{j}^{2}$. The limit on the right side exists since for any bounded Borel $\Delta$ :

$$
\begin{aligned}
& \left(\left[\oplus_{j=1}^{L} E^{j}(\Delta)\right] \int_{\Delta} \Theta_{1}^{1 \oplus \cdots \oplus L} d \theta_{L}(\lambda), \int_{\Delta} \Theta_{1}^{1 \oplus \cdots \oplus L} d \theta_{L}(\lambda)\right)= \\
& \quad=\left(\left[\oplus_{j=1}^{L} E^{j}(\Delta)\right] \mathbf{a}^{1 \oplus \cdots \oplus L}, \mathbf{a}^{1 \oplus \cdots \oplus L}\right) \leqslant\left(\left[\oplus_{i=1}^{\infty} E^{i}(\Delta)\right] \oplus_{i=1}^{\infty} a_{i}, \oplus_{i=1}^{\infty} a_{i}\right)<\infty,
\end{aligned}
$$

for all $L \in \mathbb{N}$ (Lemma 2.3). Despite seeming weak, such convergence is quite natural. Indeed, (15) implies that the cyclic subspace

$$
\mathbf{L}^{2}\left(\int_{\Delta} \Theta_{1}^{1 \oplus \cdots \oplus L} d \theta_{L}(\lambda)\right)
$$

is $\varepsilon$-close with the cyclic subspace

$$
\mathbf{L}^{2}\left(\int_{\Delta} \Theta_{1}^{1 \oplus \cdots \oplus \Lambda_{2}} d \theta(\lambda)\right)
$$


when $L$ is sufficiently big. That is, in the topology of $\mathbf{L}^{2}$ for any Borel set $\Delta$,

$$
f(T) \int_{\Delta} \Theta_{1}^{1 \oplus \cdots \oplus L} d \theta_{L}(\lambda) \rightarrow f(T) \int_{\Delta} \Theta_{1}^{1 \oplus \cdots \oplus \Lambda_{2}} d \theta(\lambda),
$$

for any Borel $f$ as $L \rightarrow \infty$. This means that

$$
\int_{\Delta} \Theta_{1}^{1 \oplus \cdots \oplus L} d \theta_{L}(\lambda) \rightarrow \int_{\Delta} \Theta_{1}^{1 \oplus \cdots \oplus \Lambda_{2}} d \theta(\lambda), \text { as } L \rightarrow \infty .
$$

(D) Define $\Omega_{3}=\left\{j \in \Omega_{1}: j=\{i, 2\}, i \in \Omega\right\}$. Construct $A_{k}: \Omega_{3}=\cup_{k=1}^{\Lambda_{3}} A_{k}$. Apply processes (B) and (C) of this proof, substituting everywhere $\Omega_{3}$ instead of $\Omega_{2}$. We obtain a vector-function $\Theta_{2}^{1 \oplus \cdots \oplus \Lambda_{3}}$, which is defined on the set $\cup_{i} P\left(e_{2}^{i}\right)$. But, as we know (see (9)), the set $s_{2}$ also includes the sets where there are non-empty superpositions of $\epsilon\left(T_{i}\right)$. Therefore, designating

$$
\begin{aligned}
\Theta_{2}^{1}=\Theta_{2}^{1 \oplus \cdots \oplus \Lambda_{3}}, \Theta_{2}^{2}=\min \{ & \left.W_{1,1,2}^{1}, W_{1,2,1}^{2}\right\}, \ldots, \\
& \Theta_{2}^{\Lambda_{2}+1}=\min \left\{\Theta_{1,1 \oplus \cdots \oplus \Lambda_{2}-1, \Lambda_{2}}^{1 \oplus \cdots \oplus \Lambda_{\Lambda-1}}, W_{1, \Lambda_{2}, 1 \oplus \cdots \oplus \Lambda_{2}-1}^{\Lambda_{2}}\right\},
\end{aligned}
$$

we may again use the process $(\mathbf{C})$ to build the vector-function $\Theta_{2}\left(\vee x_{i}, \lambda\right)$ defined on $s_{2}$ and $\Theta_{2}\left(\vee x_{i}, \lambda\right)=0$ for $\lambda \in \mathbb{R} \backslash s_{2}$. Using processes (B), (C), (D) and formula (9), we finally obtain $\Theta_{m}\left(\vee x_{i}, \lambda\right)$.

(E) The above presented constructions show, that all vector-functions $\Theta_{k}\left(\vee x_{i}, \lambda\right)$, $k=\overline{1, m}$ are the solutions of the equation $\left(\oplus_{i \in \Omega} \tau_{i}-\lambda\right) \mathbf{y}=0$, moreover they equal zero on $\mathbb{R} \backslash s_{k}$ and satisfy formulas (10) and (11).

The last thing is to prove the linear independence. In order to make the reasonings more transparent, we prove the linear independence for the special case of two vectorfunctions

$$
\Theta_{1}=W_{1,1}^{1} \oplus W_{1,2}^{2} \oplus \max \left\{W_{1,1,2}^{1}, W_{1,2,1}^{2}\right\}
$$

and

$$
\Theta_{2}=\min \left\{W_{1,1,2}^{1}, W_{1,2,1}^{2}\right\}
$$

Without loss of generality suppose that $\max \left\{W_{1,1,2}^{1}, W_{1,2,1}^{2}\right\}=W_{1,1,2}^{1}$. It is clear that

$$
\begin{aligned}
\alpha \Theta_{1}+\beta \Theta_{2}= & \\
=\alpha\left(\left\{W_{1,1}^{1}, 0,0,0\right\}+\left\{0,0, W_{1,2}^{2}, 0\right\}+\left\{0, W_{1,1,2}^{1}, 0,0\right\}\right)+\beta\left\{0,0,0, W_{1,2,1}^{2}\right\} & = \\
=\left\{\alpha W_{1,1}^{1}, \alpha W_{1,1,2}^{1}, \alpha W_{1,2}^{2}, \beta W_{1,2,1}^{2}\right\} & =0
\end{aligned}
$$

implies $\alpha=\beta=0$. The linear independence in the general case is proved using the same ideas. Thus, the linear independence is proved and this finishes the proof of the theorem.

Note, that the given proof introduces the general method of constructing eigenfunctions for a vector-operator. For theoretical purposes, the form of the obtained eigenfunctions could be simplified by totally ordering the set $\left\{T^{j}\right\}_{j=1}^{\Lambda_{2}}$. This is achieved by saying that $T^{k} \preceq T^{s}$ if $\max \left\{W_{1, k, s}^{k}, W_{1, s, k}^{s}\right\}=W_{1, k, s}^{k}$. At that, $T^{k} \simeq T^{s}$ if and only if $T^{k} \preceq T^{s}$ and $T^{s} \preceq T^{k}$. According to this, we build $\oplus_{j=1}^{\Lambda_{2}} T^{j}$, where $T^{j} \preceq T^{j+1}$, 
$j=\overline{1, \Lambda_{2}-1}$ if $\Lambda_{2} \geqslant 2$. The obtained vector-operator is obviously equivalent to the initial vector-operator (comprising unordered operators).

As an important corollary of Theorem 4.1 we obtain

THEOREM 4.2 (Eigenfunction expansions). For any $\mathbf{w} \in \mathbf{L}^{2}$, there exists a decomposition

$$
\mathbf{w}=\sum_{k=1}^{m} \lim _{n \rightarrow \infty} \int_{-n}^{+n}(U \mathbf{w})^{k}(\lambda) \Theta_{k}\left(\vee x_{i}, \lambda\right) d \theta(\lambda)
$$

Proof. From the process of building $\Theta_{k}$ in the previous proof and, in particular (4), it follows that

$$
\left(E(\Delta) F(T) \mathbf{a}^{k}\right)\left(\vee x_{i}\right)=\int_{\Delta} \Theta_{k}\left(\vee x_{i}, \lambda\right) F(\lambda) d \mu_{\mathbf{a}_{k}}(\lambda)
$$

Substituting here $F=(U \mathbf{w})^{k}$, we obtain

$$
\int_{-n}^{n} \Theta_{k}\left(\vee x_{i}, \lambda\right)(U \mathbf{w})^{k}(\lambda) d \mu_{\mathbf{a}_{k}}(\lambda)=E[-n, n] F(T) \mathbf{a}^{k} \rightarrow F(T) \mathbf{a}^{k}=U^{k^{-1}} F=\mathbf{w}^{k} .
$$

Now the statement of the theorem becomes clear, since $\mathbf{w}=\oplus_{k=1}^{m} \mathbf{w}_{k}$.

Acknowledgements. 1. The work has been carried out with the financial support of the Abdus Salam International Center for Theoretical Physics (grants AC-84 and MISC-03/14) and the Foundation for Basic Research of the Republic of Uzbekistan. 2. The author is grateful to Professor R.R. Ashurov for his productive pieces of advice and deep attention to the work; to the MAA's anonymous referees for their careful reading of the manuscript and making very useful remarks, in particular for pointing out the reference [2].

\section{REFERENCES}

[1] Gesztesy F. And KIRsch W., One dimensional Schrödinger operators with interactions singular on a discrete set, J. Reine Angew. Math., 362 (1985), pp. 28-50.

[2] Everitt W.N., Shubin C., Stolz G., Zettl A., Sturm-Liouville problems with an infinite number of interior singulrities, Spectral Theory and Computational Methods of SturmLiouville Problems (Knoxville, TN, 1996), Lecture Notes in Pure and Appl. Math., 191, Dekker, New York (1997), pp. 211-249.

[3] Everitt W.N. AND ZetTl A., Quasi-differential operators generated by a countable number of expressions on the real line, Proc. London Math. Soc., 64:3 (1992), pp. 524-544.

[4] Ashurov R.R. AND EveritT W.N., Linear operators generated by a countable number of quasi-differential expressions, Appl. Anal., 81:6 (2002), pp. 1405-1425.

[5] Ashurov R.R. AND EveritT W.N., Linear quasi-differential operators in locally integrable spaces on the real line, Proc. Roy. Soc. Edinb., 130A, (2000), pp. 671-698.

[6] Everitt W.N. And Markus L., Multi-interval linear ordinary boundary value problems and complex symplectic algebra, Mem. Am. Math. Soc., 715 (2001).

[7] Everitt W.N. AND Markus L., Infinite dimentional complex symplectic spaces, School of Mathematics and Statistics, Birmingham University, Preprint 2002/18, pp. 1-71

[8] Carlson R., Adjoint and self-adjoint differential operators on graphs, Electronic J. Diff. Eq., 1998:06, pp. 1-10.

[9] Carlson R., Nonclassical Sturm-Liouville problems and Schrödinger operators on radial trees, Electronic J. Diff. Eq., 2000:71, pp. 1-24.

[10] Kurasov P. And Stenberg F., On the inverse scattering problem on branching graphs, J. Phys. A: Math. Gen., 35 (2002), pp. 101-121.

[11] Sobhy El-Sayed I., On the essential spectra of regularly solvable operators in the direct sum spaces, Rocky Mt. J. Math., 29:2 (1999). 
[12] Sobhy El-Sayed I., On the spectra of non-selfadjoint differential operators and their adjoints in direct sum spaces, Int. J. Math. Math. Sc., 2003:9, pp. 557-574.

[13] Sokolov M.S., An abstract approach to some spectral problems of direct sum differential operators, Electronic J. Diff. Eq., 2003:75, pp. 1-6.

[14] Ashurov R.R. And Sokolov M.S., Spectral theorem for direct sum operators (in Russian), Proceedings of the XXXIII Conference on Scientific Innovations 2003. Angren State Pedagogical University, Angren, pp. 164-165.

[15] Ashurov R.R. And Sokolov M.S., On spectral resolutions of differential vector-operators, ICTP Preprint IC/2004/14.

[16] NAimark M.A., Linear differential operators, Ungar, New York 1968.

[17] Reed M. And Simon B., Methods of modern mathematical physics, Volume 1: Functional Analysis. Academic Press, New York 1972.

[18] Dunford N. And Schwartz J.T., Linear operators, Volume 2: Spectral Theory. Interscience, New York 1964. 
M. SOKOLOV 